\title{
Covid-19 e o aluno de medicina: qual a participação dos nossos internos?
}

\author{
Covid-19 and the medical student: how are our clinical clerkship students participating?
}

\author{
Jonas Ramos Sales ${ }^{1}$ (D) jonasramossales@gmail.com \\ Daniel Bezerra de Castro' (1) $\mid$ danieldecastro@rocketmail.com
}

\begin{abstract}
RESUMO
Introdução: A COVID-19, nova doença infecciosa aguda causada pelo SARS-Cov-2, descoberta na China em dezembro de 2019, é caracterizada hoje, pela OMS - como pandemia. O regime de distanciamento social implementado em todo o mundo causou importante impacto sobre vários setores, em particular o da educação. As mudanças ocorridas, no Brasil, no âmbito dos cursos de saúde de nível superior, impactaram de forma significativa o desenvolvimento das atividades de internato desempenhadas por acadêmicos de medicina.

Objetivo: O estudo buscou identificar as principais mudanças ocorridas na realização do internato dos estudantes de medicina e a visão do interno mediante tais mudanças.

Método: Foi elaborado questionário na plataforma Google Forms acerca do impacto da pandemia por COVID-19 nas atividades do internato de medicina. O universo pesquisado era composto por internos de medicina das universidades públicas e privadas de Fortaleza. Os dados coletados foram armazenados em planilhas de Excel e depois analisados no software SPSS. O teste do qui-quadrado e o de associação linear foram utilizados para avaliar a associação entre as variáveis categóricas nominais e ordinais. Em algumas comparações foi também calculado o V de Cramer. O nível de significância para todos os testes foi considerado com base no $p<0,05$.
\end{abstract}

Resultado: A amostra foi composta por 303 estudantes, dos quais 195 (64,4\%) não pararam suas atividades de internato e 108 (35,6\%) pararam tais atividades. Esses alunos utilizaram alguns motivos principais como justificativas para a paralisação ou não de suas atividades, bem como responderam, em maioria, terem tido estímulo de seus professores para escolher uma dessas duas opções. Dos que não prosseguiram com o internato, $71,3 \%$ disseram terem se sentido prejudicados com a paralisação. Além disso, uma série de correlações foram observadas a partir da comparação das respostas de alunos divididos por semestres ou por tipos de instituição (pública ou privada).

Conclusão: A pandemia por COVID-19 determinou impacto negativo na realização das atividades de internato por estudantes de medicina. Dessa forma, fica clara a necessidade por estudo de estratégias que minimizem os danos infligidos aos internos de medicina, acarretados por essa crise mundial.

Palavras-chave: Internato e Residência; Infecções por coronavírus; Pandemias.

\section{ABSTRACT}

Introduction: COVID-19, a new acute infectious disease caused by SARS-Cov-2, discovered in China in December 2019, is currently characterized by the WHO as a pandemic. The social distancing measures implemented around the world have had a major impact on various sectors, in particular, education. The changes that have occurred in Brazil in the context of higher education courses have had a significant effect on the clinical clerkship activities conducted by medical students.

Objective: The study sought to identify the main changes caused in clinical clerkships and the view of those clerkship students in light of such changes.

Method: A questionnaire was devised on Google Forms about the impact of the COVID-19 pandemic on clerkship activities. The research sample consisted of clerkship students from public and private universities in Fortaleza. The collected data were stored in Excel spreadsheets and then analyzed using the SPSS software. The chi-square test and the linear association test were used to assess the association between the nominal and ordinal categorical variables. In some comparisons Cramer's $V$ was also calculated. The level of significance for all tests was considered based on $p<0.05$.

Result: The sample consisted of 303 students, of whom 195 (64.4\%) did not stop their clerkship activities and 108 (35.6\%) did stop such activities. The students reported reasons to justify their decision to interrupt or continue the activities, and the majority responded that they had been encouraged by their teachers to choose one of those two options. Of those who did not continue with the clerkship, $71.3 \%$ said they felt the interruption had been detrimental to them. Furthermore, a series of correlations were observed by comparing the responses of students divided by semesters or by types of institution (public or private).

Conclusion: The COVID-19 pandemic has had a negative impact on clerkship activities. There is, therefore, a clear need to study strategies that minimize the damage caused by this global crisis on clerkship students.

Keywords: Clerkships and Residency; Coronavirus Infections; Pandemics.

${ }^{1}$ Universidade Estadual do Ceará, Fortaleza, Ceará, Brasil.

Editora-chefe: Rosiane Viana Zuza Diniz.

Editor associado: Roberto Zonato Esteves.

Recebido em 28/10/20; Aceito em 09/07/21.

Avaliado pelo processo de double blind review. 


\section{INTRODUÇÃO}

Coronavirus Disease 2019 (COVID-19) é uma nova doença infecciosa aguda, potencialmente grave, causada pelo SARSCoV-2, vírus da família coronavírus. Trata-se de infecção com capacidade de rápida transmissibilidade entre humanos, denotando uma grande variedade de desfechos clínicos na população, desde quadro assintomático ou respiratório leve até Síndrome Respiratória Aguda Grave (SRAG), com falência de múltiplos órgãos ou outras complicações severas ${ }^{1}$.

Os primeiros casos reportados da doença ocorreram em Wuhan (China), em dezembro de 2019, tendo sido relatados como uma pneumonia de etiologia desconhecida. Desde então, e principalmente a partir de Janeiro de 2020, apesar das medidas de controle de disseminação de infecção implementadas pelo governo chinês - incluindo quarentena - o vírus toma alcance de proporções globais². Em março de 2020, a Organização Mundial da Saúde (OMS) declara pandemia 3 .

À medida que a doença se espalha globalmente, são implementadas ações rígidas de cunho político e médicosanitarista em todo o mundo. No Brasil - em diversos estados - tais medidas se iniciaram com regras de distanciamento social, fechamento de estabelecimentos, cancelamento de eventos, até culminar, mais recentemente, com o "lockdown", legislação restritiva para a circulação de indivíduos em situações ditas não essenciais. Todas essas ações - no Brasil e no mundo - foram realizadas na tentativa de desacelerar a rápida progressão do número de casos infectados, com intuito de "achatar" a curva epidemiológica. Tais atitudes foram baseadas na experiência advinda de países pioneiros no embate ao vírus, como a China, bem como em estudos do âmbito da saúde coletiva. Assim, haveria uma menor probabilidade de esgotamento da capacidade de assistência dos sistemas de saúde ${ }^{4}$.

A introdução de tais medidas gerou profundo impacto na economia; em particular, na educação em países de todo o mundo. Em se tratando da educação no ensino superior, grande parte das instituições públicas e privadas tiveram suas atividades suspensas 5 . Estudantes e professores dos cursos de áreas da saúde, incluindo Medicina, tiveram que reformular suas práticas de ensino e de aprendizagem. Algumas mudanças foram impostas, quase que diariamente, sobre o papel desses indivíduos no período de pandemia ${ }^{6,7}$.

O questionamento sobre o papel da Medicina, particularmente do interno de medicina no enfrentamento da COVID-19, pairou sobre líderes da educação de diversas nações. Houve - em todo o período - muitas dúvidas quanto ao real benefício ou malefício da atuação desses internos no front. A American Association of Medical Colleges, por exemplo, se pronunciou contra a atuação de estudantes de medicina na assistência direta a pacientes infectados, salvo em situação emergencial, quando haveria necessidade crítica de força de trabalho no cuidado à saúde ${ }^{8}$. O American College of Physicians - em contrapartida - alega que internos de medicina, apesar de não serem funcionários nos serviços de saúde, possuem um papel claro de atuação como médicos em treinamento, não apenas como aprendizes indiretos ${ }^{9}$.

No Brasil, o Ministério da Educação; em especial, os estados, em sua autonomia, pronunciaram-se em decretos e portarias, dando liberdade de decisão às Escolas de Medicina públicas e privadas sobre a continuidade ou não das atividades de estágio obrigatório. As mesmas determinações proíbem aulas presenciais para semestres inferiores aos do internato ${ }^{10}$.

Nesse cenário, que intriga coordenadores e docentes de cursos de medicina, a proposição de uma postura concisa e segura para o desenvolvimento das atividades de internato é um grande desafio a ser superado. No contexto atual, o estudante é colocado à prova por inúmeros fatores os quais o fazem refletir não só sobre o seu real papel no enfrentamento da crise, mas também quanto à executabilidade de suas ações. Faz isso, a despeito de empecilhos ou até mesmo prejuízos à sua formação acadêmica ${ }^{11}$. Isso leva o aluno à busca de estratégias que o façam minimizar danos, o que inclui até mesmo a possibilidade de seu afastamento provisório de atividades ${ }^{8}$.

Os estudantes dos dois últimos anos da faculdade de medicina são, portanto, bastante afetados por todas as mudanças educacionais ocorridas nesse período ${ }^{12}$. Indisponibilidade de preceptores nos serviços, suspensão de atendimentos eletivos e adiantamento de graduações em medicina são apenas alguns exemplos de tais mudanças ${ }^{13-15}$. Além destes, outros impactos são observados pelos autores, mas ainda pouco descritos na literatura.

É necessário aliar a boa experiência do ensino do internato vivida por algumas faculdades (a nível local ou internacional) durante pandemia, ao que já existe de evidência científica em educação médica, atualmente ${ }^{16}$. A partir disso, é possível a montagem de uma conjectura onde o interno de medicina tenha minimizado o impacto em suas atividades tendo em vista a indiscutível importância do internato em sua formação acadêmica. Identificar as decisões tomadas pelos internos quanto ao desenrolar do seu internato no período de pandemia, bem como os fatores motivadores para essa tomada de decisões, constituem-se num passo fundamental para a tentativa de ganhos com melhoria de planejamentos ${ }^{17}$.

Os autores têm como objetivo mostrar o impacto da pandemia por COVID-19 na formação acadêmica de internos de medicina, bem como coletar e interpretar dados com a intenção de colaborar para a realização de planejamentos de internato pelas faculdades de Medicina. 
O trabalho se justifica na importância do delineamento de uma realidade mais organizada para o prosseguimento das atividades do internato de medicina a fim de proporcionar uma experiência de estágio obrigatório adequada ao acadêmico, mesmo em período de crise. Cabe lembrar que o cenário durante a pandemia é caracterizado por rodízios de internato regulados por indisponibilidade de vagas para internos de medicina nos diferentes serviços de saúde.

\section{MÉTODOS}

O presente estudo se desenvolveu a partir de uma pesquisa descritiva, de natureza quantitativa, que teve o intuito de realizar um levantamento transversal por meio da aplicação de questionário. Este, acerca do impacto da pandemia por COVID-19 na realização das atividades do internato por graduandos de medicina de universidades públicas e privadas de Fortaleza.

O universo foi constituído por internos das faculdades de medicina de Fortaleza - UECE, UFC, UNIFOR e UNICHRISTUS. Para realizar esse estudo, buscou-se a aplicação do questionário da pesquisa via mídias digitais, em especial os serviços de mensagens online gratuitas, como WhatsApp Messenger por meio de link convocando a participação de estudantes.

Ao clicar no link do questionário, o estudante era direcionado - inicialmente - ao termo de consentimento livre e esclarecido. Esse tinha o objetivo de informar aos participantes da pesquisa a natureza do trabalho, assim como assegurar-lhes que a sua participação seria voluntária e anônima. Apenas após confirmação de leitura e concordância com o termo de consentimento, o aluno poderia prosseguir com o preenchimento do questionário. Esse, por sua vez, foi construído a partir da plataforma do Google Forms, de onde foi disponibilizado o link para a participação dos interessados. Tal instrumento era composto por 18 itens cuja função era traçar o perfil do participante vinculado ao tipo de acometimento causado pela pandemia por COVID-19 nas suas atividades de internato. As perguntas diziam respeito a: sexo; idade; semestre do internato; faculdade em que cursa medicina; continuação ou não das atividades do internato; motivação para continuação; motivação para parada de atividades; frequência de estudo em domicílio; prejuízo relacionado à paralisação de atividades; estímulo do corpo docente quanto à paralisação ou continuação de atividades do internato; avaliação da preceptoria; o papel do interno nos serviços de saúde; avaliação da experiência de atuação nos serviços de saúde.

Os dados coletados foram armazenados em planilhas de Excel; depois, analisados no software SPSS, versão 23 para MAC OSX. Variáveis categóricas foram expressas como contagem absoluta e porcentagens. O teste do qui-quadrado e o de associação linear (linear-by-linear association) foram utilizados para avaliar a associação entre as variáveis categóricas nominais e ordinais. Em algumas comparações, o V de Cramer foi calculado para avaliar o grau de associação entre variáveis ordinais e os grupos. A idade foi expressa como média \pm desvio padrão e comparada entre os grupos usando o teste ANOVA. O nível de significância foi de $5 \%$ para todos os testes, considerando $\mathrm{p}<0,05$.

\section{RESULTADOS}

O perfil dos alunos do quinto e do sexto ano dos cursos de medicina que compuseram a amostra do estudo - em números absolutos - constituiu-se de 105 estudantes do sexo masculino e 198 estudantes do sexo feminino. A distribuição possui predominância de acadêmicos das universidades particulares de Fortaleza $(55,8 \%)$ e de alunos do $3^{\circ}$ semestre do internato (35\%), quando comparado aos demais semestres. A média de idade foi de 24,2 anos, com desvio padrão de 2,7 anos (Tabela 1).

Do total de 303 entrevistados, 195 (64,4\%) responderam não ter parado suas atividades de internato durante a pandemia. A principal justificativa - em $70,8 \%$ dos casos - foi a necessidade de não atrasar a data de formatura; desses - 195 alunos, $81,5 \%$ - afirmaram ter tido algum tipo de estímulo, por parte do corpo docente, para a continuação do internato. Para fins desse estudo, considerou-se como estímulo docente qualquer interação (oral ou escrita) entre aluno e professor, na qual foram mencionados, por este último - de forma ocasional e sem vínculo oficial com as decisões das coordenações de internato - benefícios quanto à continuação do internato.

Tabela 1. Estatística descritiva dos internos pesquisados.

\begin{tabular}{lcc}
\hline & Grupo total \\
\hline Idade & & $24,2 \pm 2,7$ \\
\hline Sexo & Feminino & $198(65,3 \%)$ \\
\hline Semestre do Internato & \\
& Internato 1 & $63(20,8 \%)$ \\
& Internato 2 & $75(24,8 \%)$ \\
& Internato 3 & $106(35 \%)$ \\
Internato 4 & $59(19,5 \%)$ \\
\hline Faculdade & \\
& UECE & $49(16,2 \%)$ \\
UFC & $85(28,1 \%)$ \\
UNIFOR & $87(28,7 \%)$ \\
UNICHRISTUS & $82(27,1 \%)$ \\
\hline
\end{tabular}


No entanto, quando se verificou o nível de aprendizado de conhecimentos clínicos entre os internos que optaram por prosseguir no internato, $44,1 \%$ avaliou o aprendizado como regular e $29,7 \%$ como bom. No que se refere à avaliação geral da experiência nos serviços em que os internos passaram durante a pandemia, $46,2 \%$ dos alunos consideraram a experiência boa; 35,9\%, regular. Nestes quesitos, foi utilizada a escala de Likert (ótimo, bom, regular, ruim e péssimo) como método de avaliação. Do mesmo número total de pesquisados que não pararam o internato, quando arguidos sobre o nível de importância do seu papel nos serviços em que estiveram inseridos durante a pandemia, 74,9\% afirmaram que têm papel com nível de importância auxiliar no serviço de saúde. As alternativas dadas para esse questionamento foram as seguintes: indiferente ao serviço; atrapalha no serviço; auxilia no serviço e essencial ao serviço.

O restante - 108 entrevistados (35,6\%) - respondeu ter parado suas atividades de internato durante a pandemia, utilizando como as três principais justificativas a dificuldade de aceitação de internos nos hospitais-escola (50,9\%), o medo de transmitir COVID-19 aos familiares com quem moravam $(41,7 \%)$ e o medo de contrair a referida doença (31,5\%). Destes 108 alunos, 73,1\% respondeu ter se sentido prejudicado com essa paralisação. Sessenta e dois por cento respondeu ter havido algum estímulo, por parte do corpo docente, à paralisação do internato.

Ao traçar o cruzamento de dados para estabelecer padrões de significância estatística por meio do $\mathrm{p}$ valor e do $\mathrm{V}$ de Cramer, foi possível observar algumas relações.

A Tabela 2, a seguir, mostra uma distribuição irregular dos internos quanto ao sexo e às faculdades, quando comparados os semestres do internato. No entanto, quando comparados em relação ao estímulo docente à paralisação ou continuação do internato, houve um padrão de regularidade de distribuição. O mesmo padrão pode ser observado em relação à variável de sentirem-se prejudicados com a paralisação do internato, pois não houve significância estatística, confirmado pelo $p>0,05$.

A análise do motivo que levou à parada das atividades do internato, quando comparada em relação aos semestres do internato, não mostrou significância estatística, revelado por $p>0,05$ em todas as respostas. Já o mesmo tipo de análise, quando voltada para os motivos que levaram à continuação do internato, mostrou relevância. Tal análise revelou que os semestres inferiores possuem maior valor expressivo de respostas nos itens referentes a agregarem experiência para o gerenciamento de crises ou a se ocuparem com atividades de ensino, (demonstrado pelo V de Cramer de 0,236 e 0,248 para as respectivas respostas), quando comparado com os semestres mais avançados. Os alunos do quarto semestre foram os que menos referiram, como justificativa para a continuação do internato, querer cooperar na pandemia. A resposta deles esteve concentrada, especialmente, em não atrasar a formatura (Tabela 3).

Quando questionados sobre a experiência geral vivenciada nos serviços de saúde em que se inseriram, os alunos do $3^{\circ}$ e $4^{\circ}$ semestres deram as piores notas de avaliação nesse quesito ( $p<0,001)$. Resultado semelhante foi observado com relação à avaliação do aprendizado clínico individual no período de pandemia pelos mesmos alunos mencionados $(p=0,015)$.

A Tabela 4 - abaixo - compara o perfil de respostas dos internos divididos por universidades públicas e privadas. Dessa forma, mostra que os alunos das universidades privadas

Tabela 2. Cruzamento de variáveis por semestre de internato.

\begin{tabular}{|c|c|c|c|c|c|c|}
\hline & \multicolumn{4}{|c|}{ Semestre do Internato } & \multirow[b]{2}{*}{ V de Cramer } & \multirow[b]{2}{*}{$\mathbf{p}$} \\
\hline & Internato 1 & Internato 2 & Internato 3 & Internato 4 & & \\
\hline Idade & $23,7 \pm 2,3$ & $23,4 \pm 2,1$ & $24,8 \pm 3,2$ & $24,9 \pm 2,6$ & & \\
\hline Faculdade & & & & & 0,284 & $<0,001$ \\
\hline UECE & $22(34,9 \%)$ & $0(0)$ & $26(24,5 \%)$ & $1(1,7 \%)$ & & \\
\hline UNIFOR & $16(25,4 \%)$ & $27(36 \%)$ & $22(20,8 \%)$ & $22(37,3 \%)$ & & \\
\hline UNICHRISTUS & $23(36,5 \%)$ & $15(20 \%)$ & $22(20,8 \%)$ & $22(37,3 \%)$ & & \\
\hline $\begin{array}{l}\text { Houve algum estímulo, por parte do corpo } \\
\text { docente, para a paralisação do internato }\end{array}$ & $13(61,9 \%)$ & $17(73,9 \%)$ & $25(62,5 \%)$ & $12(50 \%)$ & 0,163 & 0,414 \\
\hline $\begin{array}{l}\text { Se sente prejudicado com relação à } \\
\text { paralisação de suas atividades do internato }\end{array}$ & $16(76,2 \%)$ & $14(60,9 \%)$ & $29(72,5 \%)$ & $20(83,3 \%)$ & 0,171 & 0,37 \\
\hline
\end{tabular}


foram os que mais responderam ter parado suas atividades de internato; além disso, foram também os que mais afirmaram ter tido estímulo, pelo corpo docente, à paralisação dessas atividades. Ambos os questionamentos apresentaram $p<0,001$ na comparação entre universidades. A mesma tabela mostra altos percentuais de respostas de alunos que se sentem prejudicados com a paralisação do internato, não havendo grande variabilidade entre os perfis público e privado nesse quesito.
Quando se correlacionam os motivos atribuídos à parada das atividades com as respostas dos internos - divididos em universidades públicas e privadas - é possível observar relevância estatística em três respostas: medo de transmitir COVID-19 aos familiares, receio de prejudicar o aprendizado e falta de EPI para os internos. Os estudantes de universidades públicas foram os que mais optaram por tais repostas (Tabela 5).

Em se tratando da avaliação geral da experiência de

Tabela 3. Estatística das justificativas por semestre do internato.

\begin{tabular}{|c|c|c|c|c|c|c|}
\hline & \multicolumn{4}{|c|}{ Semestre do Internato } & \multirow{2}{*}{ V de Cramer } & \multirow{2}{*}{$\mathbf{p}$} \\
\hline & Internato 1 & Internato 2 & Internato 3 & Internato 4 & & \\
\hline \multicolumn{7}{|c|}{ Motivos pelos quais pararam as atividades do internado durante a pandemia } \\
\hline Medo de contrair a doença & $6(28,6 \%)$ & $9(39,1 \%)$ & $12(30 \%)$ & $7(29,2 \%)$ & 0,086 & 0,848 \\
\hline Medo de transmitir a doença aos familiares & $7(33,3 \%)$ & $11(47,8 \%)$ & $16(40 \%)$ & $11(45,8 \%)$ & 0,104 & 0,759 \\
\hline Receio de prejudicar o aprendizado & $8(38,1 \%)$ & $5(21,7 \%)$ & $11(27,5 \%)$ & $2(8,3 \%)$ & 0,232 & 0,12 \\
\hline $\begin{array}{l}\text { Receio de atuar em serviços com carga } \\
\text { horária sobrecarregada }\end{array}$ & $1(4,8 \%)$ & $1(4,3 \%)$ & $3(7,5 \%)$ & $2(8,3 \%)$ & 0,067 & 0,923 \\
\hline Dificuldade de meios de locomoção & $1(5 \%)$ & $1(4,3 \%)$ & $1(2,5 \%)$ & $1(4,2 \%)$ & 0,052 & 0,961 \\
\hline $\begin{array}{l}\text { Dificuldade de aceitação de internos nos } \\
\text { hospitais-escola }\end{array}$ & $12(57,1 \%)$ & $9(42,9 \%)$ & $20(50 \%)$ & $13(54,2 \%)$ & 0,096 & 0,805 \\
\hline Falta de EPI para internos nos serviços & $2(9,5 \%)$ & $6(26,1 \%)$ & $8(20 \%)$ & $3(12,5 \%)$ & 0,157 & 0,444 \\
\hline \multicolumn{7}{|c|}{ Motivos pelos quais continuaram as atividades do internato durante a pandemia } \\
\hline Por querer ajudar/cooperar na pandemia & $15(36,6 \%)$ & $22(42,3 \%)$ & $32(48,5 \%)$ & $7(20 \%)$ & 0,205 & 0,043 \\
\hline $\begin{array}{l}\text { Para se formar o mais rápido possível (não } \\
\text { atrasar a formatura) }\end{array}$ & $30(71,4 \%)$ & $38(73,1 \%)$ & $41(62,1 \%)$ & $29(82,9 \%)$ & 0,160 & 0,172 \\
\hline $\begin{array}{l}\text { Para ter experiência em gerenciamento de } \\
\text { crises }\end{array}$ & $18(42,9 \%)$ & $21(40,4 \%)$ & $26(39,4 \%)$ & $4(11,4 \%)$ & 0,236 & 0,013 \\
\hline $\begin{array}{l}\text { Para não ficar em casa sem atividades de } \\
\text { ensino }\end{array}$ & $13(36,1 \%)$ & $20(46,5 \%)$ & $25(43,1 \%)$ & $4(12,9 \%)$ & 0,248 & 0,016 \\
\hline
\end{tabular}

Tabela 4. Cruzamento de variáveis por caráter institucional.

\begin{tabular}{|c|c|c|c|}
\hline & Universidade Privada & Universidade Pública & $\mathbf{p}$ \\
\hline Idade & $24,2 \pm 2,9$ & $24,2 \pm 2,5$ & \\
\hline Sexo, masculino & $41(24,3 \%)$ & $64(47,8 \%)$ & $<0,001$ \\
\hline Internato 1 & $39(23,1 \%)$ & $24(17,9 \%)$ & \\
\hline Internato 2 & $42(24,9 \%)$ & $33(24,6 \%)$ & \\
\hline Internato 4 & $44(26 \%)$ & $15(11,2 \%)$ & \\
\hline Parou as atividades do internato durante a pandemia? & $87(51,5 \%)$ & $21(15,7 \%)$ & $<0,001$ \\
\hline $\begin{array}{l}\text { Houve algum estímulo/apoio, por parte do corpo docente do } \\
\text { curso, para a paralisação do internato }\end{array}$ & $61(70,1 \%)$ & $6(28,6 \%)$ & $<0,001$ \\
\hline $\begin{array}{l}\text { Se sente prejudicado com relação à paralisação de suas atividades } \\
\text { do internato }\end{array}$ & $63(72,4 \%)$ & $16(76,2 \%)$ & 0,726 \\
\hline
\end{tabular}


Tabela 5. Estatística das justificativas por caráter institucional

Universidade Privada Universidade Pública

Motivos pelos quais pararam as atividades do internado durante a pandemia

Medo de contrair a doença

$26(29,9 \%)$

$8(38,1 \%)$

0,467

Medo de transmitir a doença aos familiares

$30(34,5 \%)$

Receio de prejudicar o aprendizado

$17(19,5 \%)$

$15(71,4 \%)$

0,002

Receio de atuar em serviços com carga horária sobrecarregada

$4(4,6 \%)$

$9(42,9 \%)$

0,025

Dificuldade de meios de locomoção

$2(2,3 \%)$

$3(14,3 \%)$

0,106

Dificuldade de aceitação de internos nos hospitais-escola

$46(54,1 \%)$

$2(9,5 \%)$

0,172

Falta de EPI para internos nos serviços

$12(13,8 \%)$

$8(38,1 \%)$

0,118

$7(33,3 \%)$

0,035

\section{Motivos pelos quais continuaram as atividades do internato durante a pandemia}

Por querer ajudar/cooperar na pandemia

$26(32,1 \%)$

$50(44,2 \%)$

0,087

Para se formar o mais rápido possível (não atrasar a formatura)

$60(73,2 \%)$

Para ter experiência em gerenciamento de crises

$29(35,4 \%)$

$78(69 \%)$

0,530

Para não ficar em casa sem atividades de ensino

$27(36,5 \%)$

$40(35,4 \%)$

0,996

$35(37,2 \%)$

0,921

internos nos serviços que passaram durante a pandemia, pode ser observado que os alunos de universidades privadas atribuíram melhores notas (respostas "bom" e "ótimo" do questionário) a essa avaliação geral, em comparação com os alunos de universidades públicas, que atribuíram as piores notas em percentual (respostas "ruim" e "péssimo"). Tal relação possui significância estatística $(p<0,001)$. O mesmo padrão de respostas se repete no quesito avaliação da preceptoria, com melhor avaliação entre estudantes de faculdades privadas e pior, entre estudantes de faculdades públicas. Também com relevância estatística.

\section{DISCUSSÃO}

O principal objetivo dessa pesquisa foi identificar o impacto da pandemia por COVID-19 na realização das atividades do internato por alunos de medicina. Avaliamos as decisões e o posicionamento dos internos, bem como as motivações que os levaram a tomar essas decisões.

A maioria dos estudantes não pararam suas atividades do internato. O principal motivo alegado foi a necessidade de não atrasar a data programada para a formatura; esse motivo não sofreu influência estatística de nenhuma variável, configurando a principal preocupação entre os acadêmicos.

Mais de $80 \%$ dos estudantes que não pararam seu internato afirmaram ter tido estímulo de professores para tal atitude. Não houve discrepância desses resultados entre semestres do internato; tampouco, entre alunos de faculdades públicas ou privadas.

Tudo isso mostra que os internos estiveram preocupados sobretudo com a data de término do seu curso, fazendo com que buscassem se inserir nos serviços de saúde mesmo em meio ao cenário de pandemia instalado. Essa busca parece ter sido influenciada pelo corpo docente da sua universidade, a ponto de deixar dúvida entre os autores se não teria sido a influência dos professores decisiva para tal ato.

Alguns trabalhos, com resultados semelhantes, até questionam a qualidade do aprendizado advindo desse engajamento. Muitos rodízios centrais do internato (os quais exigem interação médico-paciente) foram substituídos por rodízios virtuais. Ao final da formação acadêmica desses estudantes, esses tiveram que priorizar habilidades de transparência, comunicação e bom uso de recursos online para promover um aprendizado de qualidade ${ }^{18}$.

Quando solicitado aos participantes para avaliarem a experiência geral que tiveram nos serviços em que estiveram inseridos - com base na escala de Likert - mais de $80 \%$ deram nota "bom" ou "regular". Resultado semelhante (predominância de respostas "bom" e"regular") foi observado também nos quesitos avaliação da preceptoria e aprendizado de conhecimentos clínicos. No entanto, essa mediana nos resultados foi alcançada da seguinte forma: maior peso de avaliação positiva vindo das respostas dos alunos de universidades privadas ("bom" e "ótimo" na escala de Likert) e maior peso de avaliação negativa vindo das respostas dos alunos de universidades públicas ("ruim" e "péssimo" na escala de Likert) para esses critérios. Uma explicação plausível para esse resultado é a de que os internos atuaram em locais com diferentes condições para a prática do internato na situação de pandemia: os de universidades particulares, os mais privilegiados nesse quesito. Porém, os autores questionam ainda, como justificativa dos resultados, a possibilidade de os internos de universidades privadas terem criado menor expectativa nos seus rodízios de internato durante 
a pandemia, tornando-se, dessa forma, menos exigentes na avaliação que Ihes foi proposta.

Entre os diferentes semestres, os alunos do terceiro e do quarto foram os que deram a pior avaliação para a experiência geral do internato pós-pandemia, bem como para o aprendizado específico de conhecimentos clínicos. Isso pode ser justificado pela maior experiência dos acadêmicos desses semestres com tempo de internato, quando comparam, por exemplo, serviços nos quais participaram antes da pandemia com serviços nos quais participaram durante a pandemia. Tal fato corrobora a hipótese de que a pandemia por COVID-19 impactou de forma negativa no internato de medicina. Alguns trabalhos levantam, inclusive, a pauta de como será o envolvimento desses estudantes na comunidade médica. Eles não estão tendo oportunidades de vivenciar diversas experiências engrandecedoras, seja dentro de rodízios, congressos ou cursos em geral ${ }^{19}$. A perda de algumas dessas experiências dentro de rodízios de maior interesse do acadêmico pode prejudicar a decisão por qual programa de residência médica seguir em sua vida profissional. Da mesma forma, é capaz de restringir a formação de redes de network ${ }^{20}$.

Dos 108 estudantes que pararam suas atividades de internato, mais de 70\% afirmaram sentirem-se prejudicados com essa paralisação, independentemente do semestre do internato ou do tipo de instituição de ensino (universidade pública ou privada); mais de dois terços deles são de universidades privadas. Essa discrepância pode ser justificada pelo maior estímulo que os alunos de universidades particulares tiveram, de seus professores, à não continuação do internato em relação aos acadêmicos de universidades públicas.

Os dados mostram que-desses 108 estudantes-a maioria parou o internato mais influenciada por indisponibilidade de serviço ou de garantia de segurança individual (medo de contrair a doença) e coletiva (medo de transmitir a doença) do que por falta de vontade de atuação. Isso se aplica a todos os semestres do internato de forma semelhante, o que pode ser constatado pelas justificativas dadas pelos internos para essa paralisação. Isto é, para grande parte dos internos, independente do semestre que cursam, continuar o internato deve ter sido uma opção pouco exequível, especialmente para os de universidades privadas. Os cursos de medicina de Fortaleza não conseguiram garantir o bom gerenciamento das atividades dos internos durante essa crise, estabelecendo falta de oportunidade de atuação para muitos deles.

Essa dificuldade é compartilhada por várias instituições de ensino médico ao redor do mundo ${ }^{18-21}$. Muitas delas foram obrigadas a paralisar atividades do internato devido à indisponibilidade de preceptores com tempo protegido para a orientação dos estudantes ${ }^{13}$; outras tiveram que transferir os internos para outros rodízios, por exemplo devido à suspensão de cirurgias eletivas ${ }^{14}$; e algumas delas, inclusive, adiantaram a graduação de seus alunos a fim de ofertar novos membros médicos nos cenários de atendimento à COVID-19 ${ }^{15}$. O impacto no ensino é tamanho que autores, preocupados com as mudanças causadas pela pandemia, vêm pesquisando e traçando orientações a instituições de ensino que as permita aproveitar oportunidades e minimizar perdas nesse novo contexto ${ }^{21}$.

O estudo em questão apresenta algumas limitações que merecem ser citadas. Uma delas é não poder estabelecer relação direta do impacto gerado pela pandemia na vida profissional médica dos participantes. Os resultados fazem jus, apenas, ao período de internato, alvo da pesquisa em questão.

Outra limitação é não ter conseguido comparar, de forma objetiva (com critérios), as diferentes condutas tomadas por cada universidade, quanto às suas ações frente ao internato de medicina durante a pandemia. Análise deste tipo poderia ser bastante útil para estabelecer prós e contras de cada postura; com isso, traçar estratégias unificadas - as melhores possíveis - em benefício geral dos internos de medicina. Além disso, também não foi possível avaliar o nível de importância que o estímulo docente (à paralisação ou continuação do internato) apresentou no momento em que o interno decidiu parar ou não o internato. Não podemos afirmar se este foi um fator decisivo ou não para o posicionamento dos acadêmicos de medicina.

\section{CONCLUSÃO}

A partir da análise dos dados da pesquisa, fica claro o impacto negativo da pandemia por COVID-19 nas atividades de internato dos acadêmicos de medicina. Ainda que, por diferentes motivos e - principalmente - com diferentes intensidades, a maioria dos internos referem ter se sentido prejudicados, academicamente, em algum momento durante essa crise mundial. Mesmo assim, boa parte deles optou por não parar suas atividades, identificando interesses individuais e coletivos como justificativa em diferentes proporções. Muito ainda necessita ser estudado e aprendido com esse cenário global para que mais estratégias possam ser traçadas a fim de viabilizar uma minimização desse tipo de dano em períodos de crise.

\section{CONTRIBUIÇÃO DOS AUTORES}

Jonas Ramos Sales: Concepção e desenho da pesquisa, revisão de literatura, metodologia, interpretação dos resultados e formatação do artigo. Daniel Bezerra de Castro: Orientação da pesquisa, revisão da metodologia, da análise de dados e revisão do artigo.

\section{CONFLITO DE INTERESSES}

Declaramos não haver conflito de interesses. 


\section{FINANCIAMENTO}

\section{Declaramos não haver financiamento.}

\section{REFERÊNCIAS}

1. Lian J, Jin X, Hao S, Cai H, Zhang S, Zheng L, et al. Analysis of Epidemiological and Clinical features in older patients with Corona Virus Disease 2019 (COVID-19) out of Wuhan. Clin Infect Dis. 2020;1-8.

2. Chen N, Zhou M, Dong X, Qu J, Gong F, Han Y, et al. Epidemiological and clinical characteristics of 99 cases of 2019 novel coronavirus pneumonia in Wuhan, China: a descriptive study. Lancet. 2020;395:507-13.

3. World Health Organization W. WHO Director-General's opening remarks at the media briefing on COVID-19. WHO Dir Gen speeches. 2020. [acesso em 10 jul 2020]. Disponível em: https://www.who.int/dg/speeches/detail/ who-director-general-s-opening-remarks-at-the-media-briefing-oncovid-19---11-march-2020.

4. Werneck GL, Carvalho MS. A pandemia de COVID-19 no Brasil: Crônica de uma crise sanitária anunciada. Cad Saude Publica. 2020;36(5):1-4.

5. The New York Times. Colleges and universities cancel classes and move online amid coronavirus fears. 2020 [acesso em 9 jul 2020]. Disponível em: https://www.nytimes.com/2020/03/10/us/coronavirus-closings.html.

6. Roberts C. How medical education can help in a COVID-19 crisis. Clin Teach. 2020;17(3):241-3.

7. Rodrigues BB, Cardoso RRJ, Peres CHR, Marques FF. Aprendendo com o Imprevisível: Saúde Mental dos Universitários e Educação Médica na Pandemia de Covid-19. Rev Bras Educ Med. 2020;44(suppl 1):8-12.

8. Whelan A, Prescott JE, Young G, Catanese VM, McKinney R. Guidance on Medical Students' Participation in Direct Patient Contact Activities. 2020;1-6 [acesso em 12 jul 2020]. Disponível em: https://www.aamc.org/ media/42776/download.

9. Miller DG, Pierson L, Doernberg S. The Role of Medical Students During the COVID-19 Pandemic. Ann Intern Med. 2020;(April):19-21.
10. Fortaleza (Ceará). Decreto n 33.532, de 30 de março de 2020. Dispõe sobre as medidas adotadas pelo estado do Ceará para contenção do avanço do novo coronavírus, e dá outras providências. Diário Oficial do Estado do Ceará. 30 mar 2020; Série 3.

11. Koven S. We signed up for this. N Engl J Med. 2020:1-2.

12. Baker DM, Bhatia S, Brown S, Cambridge W, Kamarajah SK, McLean KA et al. Medical student involvement in the COVID-19 response. Lancet 2020;395:1254.

13. Goldenberg MN, Hersh DC, Wilkins KM, Schwartz ML. Suspending Medical Student Clerkships Due to COVID-19. Med Sci Educ. 2020;30(3):1273-6.

14. Kim CS, Lynch JB, Cohen S, Neme S, Staiger TO, Evans L, et al. One academic health system's early (and Ongoing) experience responding to COVID-19: Recommendations from the initial epicenter of the pandemic in the United States. Acad Med. 2020;95(8):1146-8.

15. Rose S. Medical Student Education in the Time of COVID-19. JAMA - J Am Med Assoc. 2020;323(21):2131-2.

16. Garcia PDW, Massarotto P, Auinger $K$, Schuepbach RA, Klinzing S. Students Supporting Critical Care - A contention plan to prevent the decompensation of ICUs in the COVID-19 pandemic:Translating Bjorn Ibsens' polio-lessons to modern times. Crit Care. 2020;24(1):1-2.

17. Choi B, Jegatheeswaran L, Minocha A, Alhilani M, Nakhoul M, Mutengesa E. The impact of the COVID-19 pandemic on final year medical students in the United Kingdom: a national survey. BMC Med Educ. 2020;20(1):206.

18. Theoret C, Ming X. Our education, our concerns: The impact on medical student education of COVID-19. Med Educ. 2020;54(7):591-2.

19. Ferrel MN, Ryan JJ. The Impact of COVID-19 on Medical Education. Cureus. 2020;12(3):10-3.

20. Akers A, Blough C, lyer MS. COVID-19 Implications on Clinical Clerkships and the Residency Application Process for Medical Students. Cureus 2020;12(4).

21. Triemstra JD, Haas MRC, Bhavsar-Burke I, Gottlieb-Smith R, Wolff M, Shelgikar AV, et al. Impact of the COVID-19 Pandemic on the Clinical Learning Environment. Acad Med. 2021. 